\title{
Random amplified polymorphic DNA-based molecular heterogeneity analysis of Salmonella enterica isolates from foods of animal origin
}

\author{
Surendra Singh Shekhawat ${ }^{1}$, Abhishek Gaurav ${ }^{1}$, Bincy Joseph², Hitesh Kumar ${ }^{1}$ and Nirmal Kumar
}

1. Department of Veterinary Public Health and Epidemiology, College of Veterinary and Animal Science, Navania, Vallabhnagar, Udaipur, Rajasthan, India; 2. Department of Veterinary Microbiology, College of Veterinary and Animal Science, Navania, Vallabhnagar, Udaipur, Rajasthan, India.

Corresponding author: Surendra Singh Shekhawat, e-mail: apsss14@gmail.com

Co-authors: AG: gaurav.vets@gmail.com,BJ: dr.binzy@gmail.com, HK: hiteshkumar10121988@gmail.com, NK: dr.nirmalvet@gmail.com

Received: 29-08-2018, Accepted: 03-12-2018, Published online: 26-01-2019

doi: 10.14202/vetworld.2019.146-154 How to cite this article: Shekhawat SS, Gaurav A, Joseph B, Kumar H, Kumar N (2019) Random amplified polymorphic DNA-based molecular heterogeneity analysis of Salmonella enterica isolates from foods of animal origin, Veterinary World, 12(1): 146-154.

\begin{abstract}
Aim: This study aims to study the significance of random amplified polymorphic DNA (RAPD) typing in heterogeneity analysis of Salmonella serovars, isolated from foods of animal origin.

Materials and Methods: Salmonella serovars isolated and identified from different foods of animal origin such as meat, milk, and egg by standard bacteriological methods. DNA isolated from all 10 isolates which are confirmed by biochemical and serotyping methods and then RAPD was performed using the primers OPB 10, primer 1290, NSC I, NSC II, and primer 3. Then, RAPD data were analyzed using the BioNumerics software, Belgium, Germany.

Results: RAPD polymerase chain reaction (PCR) using five primers, namely OPB 10, primer 1290, NSC I, NSC II, and primer 3, classified the 10 isolates into 9, 10, 10, 7, and 10 RAPD-PCR types with discriminating powers of $0.1987,0.423$, $0.50889,0.1842$, and 0.2582 , respectively. The phylogram constructed with NSC I profile classified isolates based on geographical origin. Primer 1290, NSC II, and primer 3 produced some uniform bands in all isolates indicating their binding ability in conserved genomic region. This study revealed that RAPD profile can be best used for finding out the heterogeneity at molecular level of Salmonella isolates in combination with other molecular and phenotypic typing techniques. Thus, our results support earlier observation of its significance by different workers on different Salmonella serotypes.
\end{abstract}

Conclusion: Repeatability of RAPD-PCR is insufficient to distinguish genetic differences among Salmonella serovars.

Keywords: Salmonella, random amplified polymorphic DNA, foods of animal origin, phylogram.

\section{Introduction}

Foodborne diseases are a serious a public health concern in food industry and Salmonella organisms are most frequently isolated bacterial agents of foodborne outbreaks [1]. Gram-negative Salmonella is a major health problem worldwide that causes typhoidal and non-typhoidal salmonellosis. Typhoidal and non-typhoidal illnesses cause millions of cases yearly with significant economic losses and even human deaths [2]. Most cases of non-typhoidal Salmonella (NTS) disease are associated with consumption of contaminated foods of the animal origin, particularly poultry meat and in some instances vegetables $[3,4]$. Salmonella serovars are responsible for infections occurring in developing as well as developed world and have been a major concern in terms of economic burden due to high morbidity [5]. Salmonella is an

Copyright: Shekhawat, et al. Open Access. This article is distributed under the terms of the Creative Commons Attribution 4.0 International License (http://creativecommons.org/licenses/ by/4.0/), which permits unrestricted use, distribution, and reproduction in any medium, provided you give appropriate credit to the original author(s) and the source, provide a link to the Creative Commons license, and indicate if changes were made. The Creative Commons Public Domain Dedication waiver (http:// creativecommons.org/publicdomain/zero/1.0/) applies to the data made available in this article, unless otherwise stated. important cause of foodborne (alimentary) health problems in humans [6]. Salmonella was the second laboratory confirmed etiological agent accounting for $229(30 \%)$ reported food poisoning outbreaks in the United States [7].

The development of polymerase chain reaction (PCR) technology has allowed the specific amplification of particular target segments of DNA. Several PCR-based assays have been developed for rapid detection of Salmonella spp. [8]. Serotyping is most widely used phenotypic method, but it fails to provide appropriate information due to complex serotyping scheme and lack of comparison among different laboratories, thereby limiting its application to the reference laboratories only [9]. Genotypic characterization of bacteria which are foodborne like Salmonella is very important to determine the genetic diversity of strains. Salmonella is a diverse group of bacteria with a large number of serotypes and strains present in various hosts which include animal, birds, and humans. Moreover, due to diverse ecological habitats of this bacterium, there is a need of comparing the strains from different sources to determine the clonal variation or similarity for physiological studies. Genotypic characterization helps to understand the complete 
epidemiology of the disease and aids in understanding the evolutionary pathways of various strains originating from different ecological niches. Therefore, various novel genotyping methods such as variable number tandem repeats (VNTR), multilocus VNTR, multilocus sequence typing, and ERIC-PCR have been developed and utilized [10-13] to delineate epidemiological relationships among various isolates even within the same phage types [14]. However, the search continues for the easy to use efficient method capable of differentiating strains of similar phenotype [15]. The application of random amplified polymorphic DNA (RAPD) analysis based on random amplification of genomic DNA fragments through short arbitrarily designed primers is an attractive alternative and has the potential to detect polymorphism throughout the entire genome as compared to other techniques [16].

In the present study, we tried to analyze the significance of RAPD typing in heterogeneity analysis of Salmonella serovars, isolated from foods of animal origin.

\section{Materials and Methods}

\section{Ethical approval}

In the present investigation, we have not used any live animals, therefore; no ethical approval was needed for the present study.

\section{Bacteria}

A total of 10 Salmonella isolates obtained from foods of animal origin [17] were used in the study. All the 10 positive Salmonella isolates were sent to Central Research Institute (CRI), Kasauli, Himachal Pradesh, for further serotyping.

\section{DNA isolation}

DNA isolated from all the 10 biochemically confirmed isolates using HiMedia TM Bacterial Genomic DNA Purification Kit following the manufacturer's instructions supplied along with the kit with suitable modifications. Briefly, $1.5 \mathrm{ml}$ of overnight broth culture was pelleted by centrifugation at $15,000 \mathrm{rpm}$ for $2 \mathrm{~min}$. The supernatant was discarded, and pellet was resuspended in $180 \mu 1$ of lysis solution AL. After adding $200 \mu 1$ lysis solutions $C_{1}$, it was vortexed for $15 \mathrm{~s}$ and then incubated at $55^{\circ} \mathrm{C}$ for $10 \mathrm{~min}$. Then, $200 \mu \mathrm{l}$ of ethanol $(95-100 \%)$ was added to the lysate and mixed thoroughly by vortexing for $15 \mathrm{~s}$. The lysate so obtained was transferred into spin column and centrifuged at 10,000 rpm for $1 \mathrm{~min}$. The flow-through liquid was discarded and placed in a new $2 \mathrm{ml}$ collection tube. Then, $500 \mu 1$ of prewash solution was added to the spin column and centrifuged at $10,000 \mathrm{rpm}$ for $1 \mathrm{~min}$. The flow-through was again discarded, and same collection tube was used. A volume of $500 \mu \mathrm{l}$ of diluted wash solution was added to column and centrifuged at 15,000 rpm for $3 \mathrm{~min}$ and spin again at same speed for the additional 1 min to dry the column. The HiElute spin column was placed on a fresh tube and $100 \mu 1$ of elution buffer which was kept in a water bath at $65^{\circ} \mathrm{C}$ for $30 \mathrm{~min}$. The column was incubated at room temperature for 5 min followed by centrifugation at 10,000 rpm for $1 \mathrm{~min}$. The spin column was then removed, and the collected DNA was stored at $-20^{\circ} \mathrm{C}$ for further use. The concentration of DNA isolated was estimated spectrophotometrically using Biospectrometer (Eppendorf, USA) using the following formula: DNA concentration $(\mu \mathrm{g} / \mu \mathrm{l})=\left[\mathrm{OD}_{260}\right.$ $\times$ dilution factor $\times 50 \mathrm{mg} / \mathrm{ml}] / 1000$. The purity was checked as ratio of $\mathrm{OD}_{260}$ and $\mathrm{OD}_{280}$, and the integrity of the purified DNA was assessed by running it in $0.7 \%$ agarose gel.

\section{RAPD-PCR}

RAPD-PCR was performed using genomic DNA of Salmonella isolates as a template with the random primers (Xcelris) mentioned in Table-1 [18-20]. Each PCR mixture consisted of $1 \mu \mathrm{g}$ of template DNA, $2 \mathrm{pM}$ solution of each primer (Xcelris, India), $5 \mu 110 \times$ PCR buffer (Thermo Scientific, USA), $3 \mathrm{mM} \mathrm{MgCl}$ (Thermo Scientific, USA), $300 \mu \mathrm{M}$ each nucleotides (Thermo Scientific, USA), and 3.75 units of Taq DNA polymerase (Thermo Scientific, USA) in $50 \mu \mathrm{l}$ PCR reaction mix. PCR program for NSC I primer included initial denaturation at $94^{\circ} \mathrm{C}$ for $5 \mathrm{~min}$, followed by 40 cycles of denaturation $\left(94^{\circ} \mathrm{C}\right.$ for $\left.1 \mathrm{~min}\right)$, annealing $\left(25^{\circ} \mathrm{C}\right.$ for $\left.45 \mathrm{~s}\right)$, and extension $\left(72^{\circ} \mathrm{C}\right.$ for $\left.1 \mathrm{~min}\right)$. Final extension was carried out at $72^{\circ} \mathrm{C}$ for $7 \mathrm{~min}$. For primers NSC II, 1290, OPB-10, and primer 5, PCR reaction mix and cycling conditions were same with the exception of their annealing temperature which was adjusted at $29^{\circ} \mathrm{C}, 27^{\circ} \mathrm{C}, 30^{\circ} \mathrm{C}$, and $27^{\circ} \mathrm{C}$ respectively. PCR products were characterized by submarine gel electrophoresis on $0.9 \%$ agarose gel. After electrophoresis, the gel was visualized in gel documentation system, and picture was taken. $100 \mathrm{bp}$ DNA ladder (Thermo Scientific, USA) and $1 \mathrm{~kb}$ DNA ladder (Thermo Scientific, USA) were used as molecular weight markers.

\section{Analysis of RAPD data}

The banding patterns from RAPD were analyzed with BioNumerics Software version 7.5 (Applied

Table-1: Sequences of different primers used for RAPD analysis of Salmonella enterica isolates.

\begin{tabular}{lllll}
\hline Target genes & $\begin{array}{l}\text { Primer sequence } \\
\left(\mathbf{5}^{\prime} \longrightarrow \mathbf{3}^{\prime}\right)\end{array}$ & Melting temperature $\left.\mathbf{(}^{\circ} \mathbf{C}\right)$ & Type & References \\
\hline OPB-10 & 5'-CGT CTG GGA C-3' & 34 & RAPD & {$[18]$} \\
Primer 1290 & 5'-GTG GAT GCG A-3' & 32 & RAPD & {$[19]$} \\
NSC I & 5'-AGG ACC AGG-3' & 30 & RAPD & {$[19]$} \\
NSC II & 5'-AGG GCC CGG G-3' & 34 & RAPD & {$[19]$} \\
Primer 3 & 5'-CGT GCA CGC-3' & 32 & RAPD & {$[20]$} \\
\hline
\end{tabular}


Maths, Ghent, Belgium). The gel images of respective genotypic technique were uploaded as tiff format. The gel image of the genotyping PCR was processed in four steps, namely strip creation, calculation of densitometric curves, normalization of gel image, and detection of bands on the gel. The strip creation step identifies individual lane of the sample and defines the area of it to cut from the main gel. In the densitometric step, the software builds the peaks on the basis of intensity of bands and detects the strength of the positivity of each band. The normalization step helps to save the changes in the edited image and helps to set a reference marker. Finally, in the band calculation step, each of the bands identified on the gel is compared against the reference marker and compared with other bands present on the gel. After processing the gel image, each of the lane data was added to the database of BioNumerics Software version 7.5, in particular, genotyping PCR technique. The data were used to make a comparison of the lanes for the creation of dendrogram by similarity matrix using the Dice coefficient with optimization of $1 \%$. The dendrograms were obtained by means of Unweighted Pair Group Method with Arithmetic Average clustering algorithm.

The numerical index of discrimination (D) of each primer was also calculated using the Simpson's index of diversity [21] using the following formula:

$$
\mathrm{D}=1-\frac{1}{\mathrm{~N}(\mathrm{~N}-1)} \sum_{\mathrm{j}=1}^{\mathrm{s}} \mathrm{n}_{\mathrm{j}}\left(\mathrm{n}_{\mathrm{j}}-1\right)
$$

Table-2: Details of the serotype of Salmonella enterica isolates.

\begin{tabular}{lll}
\hline Isolate No & Serotype & Source \\
\hline S98 & Salmonella Lindenburg & Chicken \\
S115 & Salmonella Lindenburg & Chicken \\
S158 & Salmonella Lindenburg & Chicken \\
S221 & Salmonella Rough & Chevon \\
S306 & Salmonella Rough & Egg \\
S410 & Salmonella Enteritidis & Egg \\
S453 & Salmonella Lindenburg & Chicken \\
S485 & Salmonella Rough & Milk \\
S522 & Salmonella Rough & Milk \\
S570 & Salmonella Typhimurium & Milk \\
\hline
\end{tabular}

Where $\mathrm{N}$ is the total number of strains in the sample population, $\mathrm{s}$ is the total number of types described, and $\mathrm{n}$ is the number of strains belonging to $j^{\text {th }}$ type.

\section{Results and Discussion}

Nowadays, advances in molecular biological tools utilizing various typing techniques make it simpler to differentiate isolates of Salmonella serovars. Keeping this in view, the attempts were made to evaluate the molecular heterogeneity among Salmonella isolates from foods of animal origin using RAPDPCR. Salmonella enterica isolates obtained from foods of animal origin were subjected to serotyping at CRI, Kasauli, Himachal Pradesh, India. The results of serotyping are presented in Table-2.

The application of RAPD analysis [22-24] based on random amplification of genomic DNA fragments through short arbitrarily designed primers allows one to start a blind walk through whole genomic DNA of an organism. The discriminatory power of this typing method can be enhanced by the use of more than one primer. For this reason, this study incorporated five random primers for differentiating isolates of Salmonella species. It was observed that all five primers employed were capable of elucidating polymorphic amplification patterns in all isolates.

RAPD-PCR using primer OPB-10 was successfully able to produce fingerprints of all the 10 isolates of Salmonella spp. tested (Figure-1). Some bands are common in all the isolates indicating the ability of this primer to bind to some conserved regions of Salmonella genomic DNA. On the basis of dendrogram prepared using RAPD-PCR assay, 10 isolates of Salmonella could be divided into nine RAPD-PCR types (Figure-2). A maximum number of bands (10) produced by Salmonella Rough-1 and minimum number of bands (4) produced by Salmonella Enteritidis isolates. The analysis of fingerprints in the dendrogram displayed that four isolates of Salmonella Lindenburg showed three different types of fingerprints, hence, dividing them into three clonal groups. This indicates the ability of OPB-10 primer to discriminate within the serovar Lindenburg also.

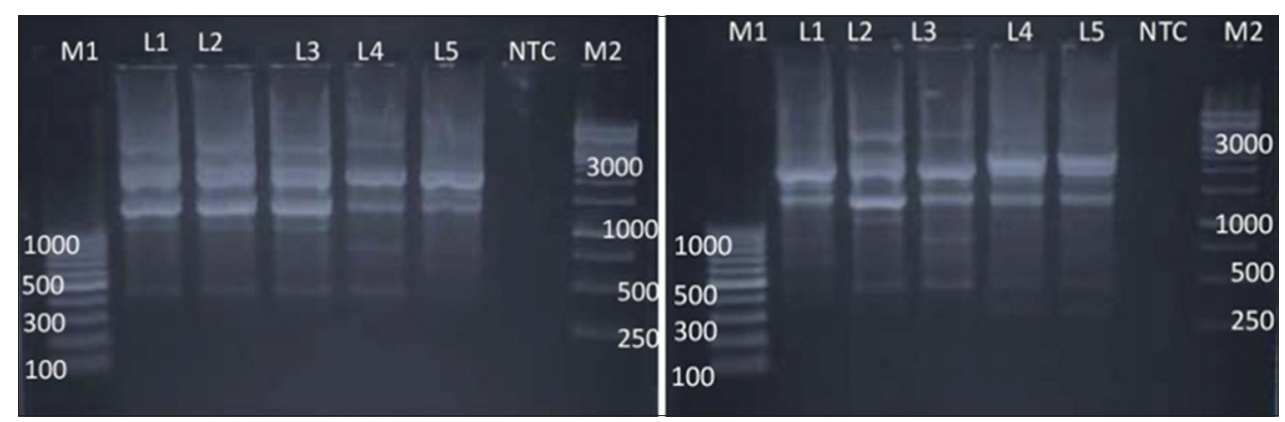

Figure-1: Random amplified polymorphic DNA- polymerase chain reaction profiles of Salmonella enterica isolates with OPB 10. Plate 1- Lane M1: 100 bp ladder; Lane 1: Salmonella Lindenburg; Lane 2: Salmonella Lindenburg; Lane 3: Salmonella Lindenburg; Lane 4: Salmonella Rough; Lane 5: Salmonella Rough; Lane 6: NTC; Lane M2:1 kb ladder and Plate 2 - Lane M1: 100bp ladder; Lane 1: Salmonella Enteritidis; Lane 2: Salmonella Lindenburg; Lane 3: Salmonella Rough; Lane 4: Salmonella Rough; Lane 5: Salmonella Typhimurium; Lane 6: NTC; Lane M2: 1 kb ladder. 
Similarly, Sumithra et al. [25] reported the high discriminative power OPB 10 primer to discriminate between the isolates of Salmonella Typhimurium. We observed an average genetic similarity between the four isolates of Salmonella Lindenburg (69.3\%). The four Salmonella Rough isolates were separated into four different RAPD types with average genetic similarity of $33.9 \%$. The genetic similarity between serotype Salmonella Lindenburg and Salmonella Enteritidis was $33.3 \%$ (66.7\% average genetic diversity). The genetic similarity between serotype Salmonella Lindenburg and Salmonella Typhimurium was $33.9 \%(66.1 \%$ average genetic diversity). The genetic similarity between serotype Salmonella Enteritidis and Salmonella Typhimurium was $33.3 \%$ (66.7\% average genetic diversity). The Salmonella isolates from chicken showed an average similarity of $69.3 \%$ (30.7\% average genetic diversity). Genetic similarity between Salmonella spp. isolated from milk ranged 33.9$71.4 \%$ with an average similarity of $52.65 \%(47.35 \%$ average genetic diversity). Average genetic similarity between Salmonella spp. isolated from egg was $33.3 \%(66.7 \%$ average genetic diversity). Average similarity between Salmonella spp. isolated from egg and chicken is $33.3 \%$ (66.7\% average genetic diversity). The discrimination power of OPB 10 was calculated as 0.1987 . Our results were contrary to the reports of Sumithra [26] who reported that OPB 10 primer has a better discrimination power $(0.88312)$ to discriminate between different isolates of Salmonella Typhimurium. As per the results obtained in this study, the discriminative power of OPB-10 primer between the different serovars is very poor.

RAPD-PCR using primer 1290 was successfully able to produce fingerprints of all the 10 isolates of Salmonella spp. tested (Figure-3). Here also, most of the bands are commonly indicating the ability of this primer to bind to the conserved regions of Salmonella. On the basis of dendrogram prepared using RAPDPCR assay, 10 isolates of Salmonella could be divided into 10 RAPD-PCR types (Figure-4). The analysis of fingerprints in the dendrogram of four isolates of Salmonella Lindenburg showed four different types of fingerprints, hence, dividing them into four clonal groups. We observed an average genetic similarity between four isolates of Salmonella Lindenburg to be $56.5 \%$ ( $43.5 \%$ average genetic diversity). The four Salmonella rough strains were separated into four different RAPD types with an average genetic similarity of $39.8 \%(60.2 \%$ average genetic diversity). The genetic similarity between serotype Salmonella Lindenburg and Salmonella Enteritidis was 50.9\% (66.7\% average genetic diversity). The level of

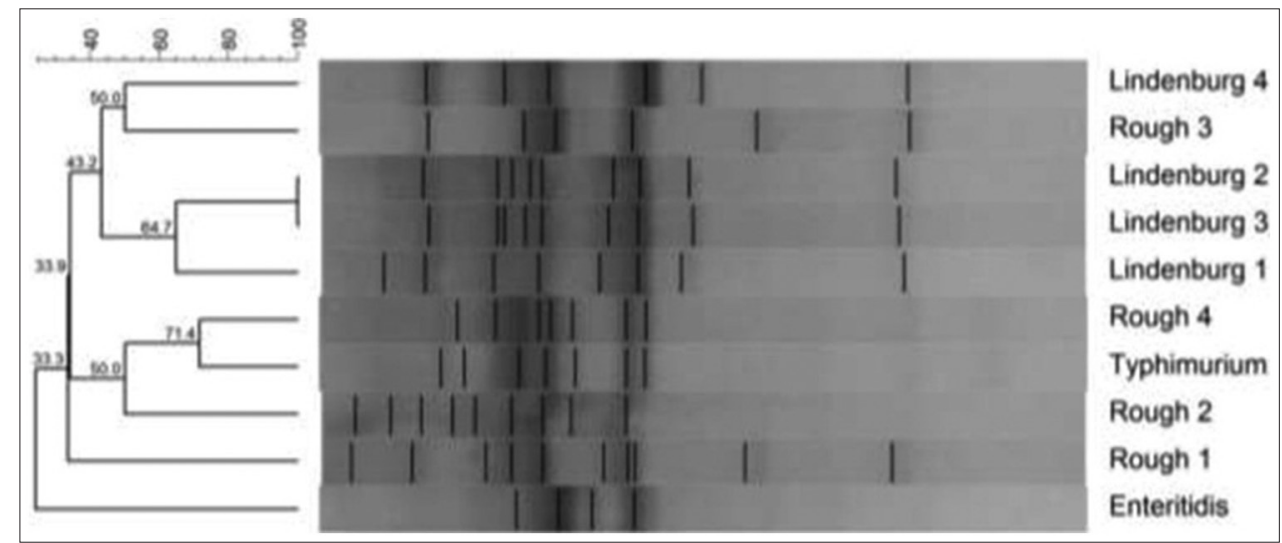

Figure-2: Dendrogram of random amplified polymorphic DNA - polymerase chain reaction profile of Salmonella enterica isolates with OPB 10.

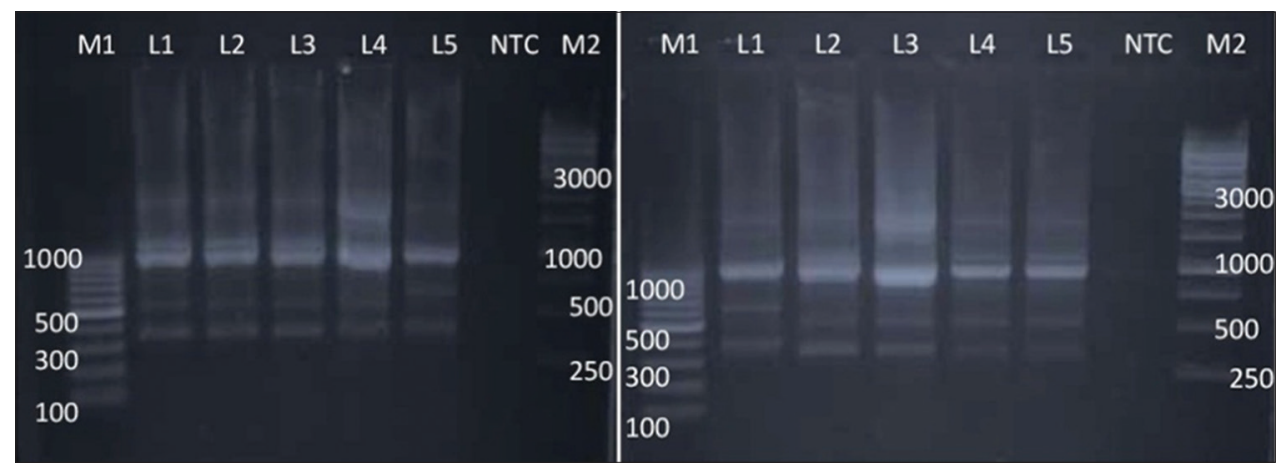

Figure-3: Random amplified polymorphic DNA - polymerase chain reaction profiles of Salmonella enterica isolates with primer 1290. Plate 1 Lane M1: 100 bp ladder; Lane 1: Salmonella Lindenburg; Lane 2: Salmonella Lindenburg; Lane 3: Salmonella Lindenburg; Lane 4: Salmonella Rough; Lane 5: Salmonella Rough; Lane 6: NTC; Lane M2: 1kb ladder and Plate 2-5: Salmonella Typhimurium; Lane 6: NTC: Lane M2: 1 kb ladder. 
similarity ranged between $43.2 \%$ and $58.6 \%$. The genetic similarity between serotype Salmonella Lindenburg and Salmonella Typhimurium was 33.9\% (66.1\% average genetic diversity). The level of similarity ranged between 43.2 and 48.3. The genetic similarity between serotype Salmonella Enteritidis and Salmonella Typhimurium was $42.3 \%$ (57.7\% average genetic diversity).

The genetic similarity among Salmonella spp. from chicken ranged between $42.3 \%$ and $72.7 \%$ with an average similarity of $56.5 \%$ (43.5\% average genetic diversity). Average similarity between Salmonella spp. isolated milk ranged from 43.2 to $58.6 \%$. Average genetic similarity between Salmonella spp. isolated from egg was $21 \%$ ( $79 \%$ average genetic diversity) and the similarity between isolates from egg and chicken was $34.65 \%$. The Simpson diversity index of primer 1290 was 0.423 .

RAPD-PCR using NSC I also produce fingerprints of all the 10 isolates of Salmonella spp. tested (Figure-5). On the basis of dendrogram prepared, 10 isolates of Salmonella could be divided into seven RAPD-PCR types (Figure-6). The analysis

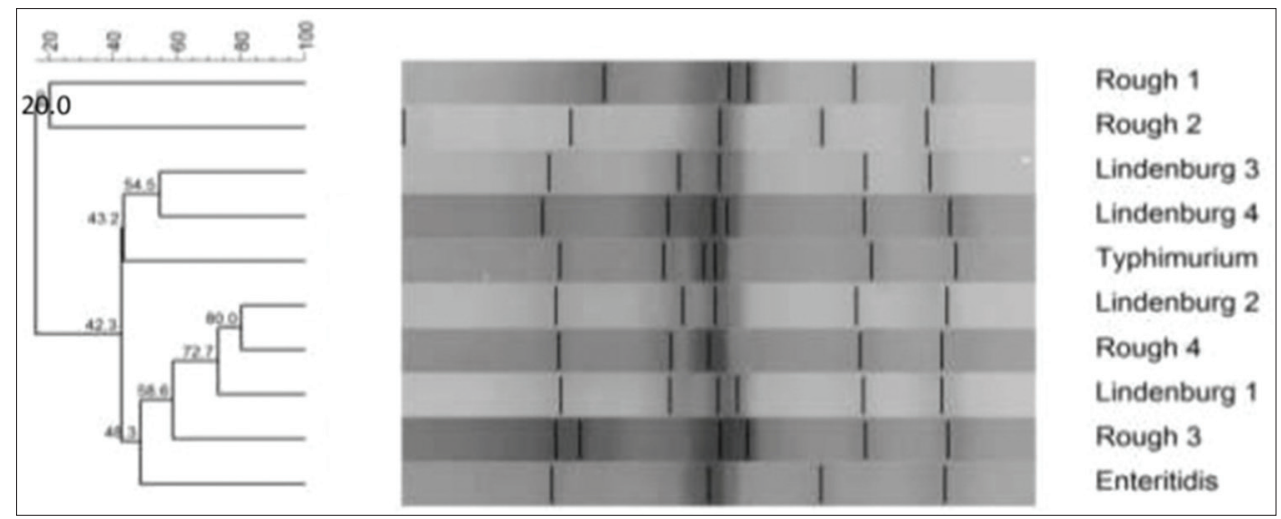

Figure-4: Dendrogram of random amplified polymorphic DNA - polymerase chain reaction profile of Salmonella enterica isolates with primer 1290 .

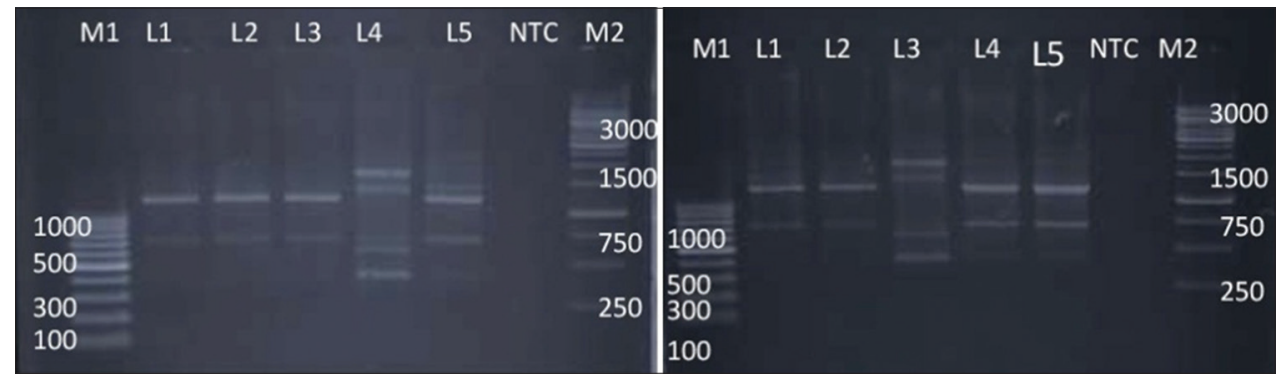

Figure-5: Random amplified polymorphic DNA - polymerase chain reaction profiles of Salmonella enterica isolates with NSC I. Plate 1-Lane M1: 100 bp ladder; Lane 1: Salmonella Lindenburg; Lane 2: Salmonella Lindenburg; Lane 3: Salmonella Lindenburg; Lane 4: Salmonella Rough; Lane 5: Salmonella Rough; Lane 6: NTC; Lane M2: 1 kb ladder and Plate 2 Lane M1: 100 bp ladder; Lane 1: Salmonella Enteritidis; Lane 2: Salmonella Lindenburg; Lane 3: Salmonella Rough; Lane 4: Salmonella Rough; Lane 5: Salmonella Typhimurium; Lane 6: NTC; Lane M2: 1 kb ladder.

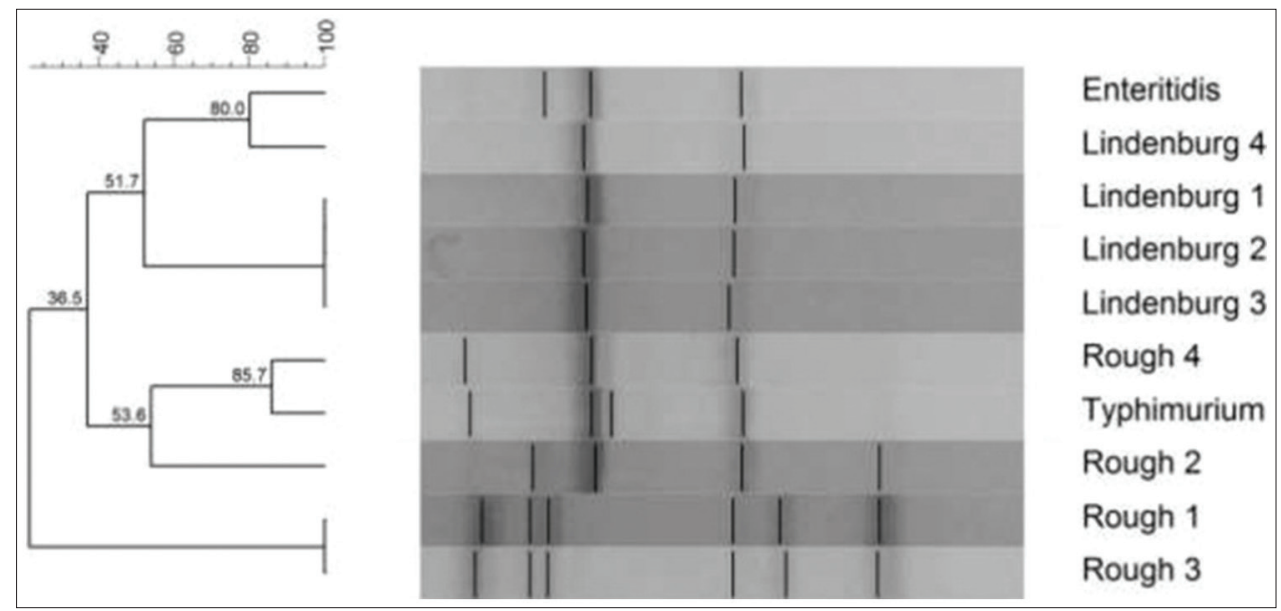

Figure-6: Dendrogram of random amplified polymorphic DNA - polymerase chain reaction profile of Salmonella enterica isolates with NSCI. 
of fingerprints in the dendrogram of four isolates of Salmonella Lindenburg showed two different types of fingerprints, hence, dividing them into two clonal groups. We observed an average genetic similarity between four isolates of Salmonella Lindenburg, $75.85 \%$ (24.15\% average genetic diversity). The level of similarity ranged between $51.7 \%$ and $100 \%$. The four Salmonella Rough strains were separated into three different RAPD types with average genetic similarity of $68.25 \%$ (31.75\% average genetic diversity). The genetic similarity between serotype Salmonella Lindenburg and Salmonella Enteritidis was $65.85 \%$ (34.15\% average genetic diversity). The level of similarity ranged between $51.7 \%$ and $80 \%$. The genetic similarity between serotype Salmonella Lindenburg and Salmonella Typhimurium was 51.7\% (48.3\% average genetic diversity) and between serotype Salmonella Enteritidis and Salmonella Typhimurium was $36.5 \%$ (63.5\% average genetic diversity). The discrimination power of NSC I was 0.50889 .

The genetic similarity among Salmonella spp. from chicken showed a genetic similarity ranged between $51.7 \%$ and $100 \%$ with an average similarity of $75.85 \%(24.15 \%$ average genetic diversity). Average similarity between Salmonella spp. isolated from milk was $62 \%$ (38\% average genetic diversity) and the similarity between the isolates from egg was $36.5 \%$ (63.5\% average genetic diversity). Average similarity between Salmonella spp. isolated from egg and chicken is $58.25 \%$ ( $41.75 \%$ average genetic diversity). This study point toward the power of NSC I primer to some extent group the Salmonella isolates based on their host origin. Sumithra et al. [25] also reported the ability of NSC I primer to cluster Salmonella Typhimurium isolates based on geographical origin. The potential ability of NSC I primer to discriminate Salmonella Gallinarum based on their geographical origin was also reported by Habtamu et al. [19].

RAPD-PCR using NSC II was also able to produce fingerprints of all the 10 isolates of Salmonella spp. tested (Figure-7). On the basis of dendrogram prepared using RAPD-PCR assay, 10 isolates of Salmonella could be divided into 10 RAPD-PCR types (Figure-8). The analysis of fingerprints in the dendrogram of four isolates of Salmonella Lindenburg showed four different types of fingerprints, hence, dividing them into four clonal groups. We observed an average genetic similarity between four isolates of Salmonella Lindenburg, $61.56 \%$ (38.44\% average genetic diversity). The four Salmonella Rough strains were separated into four different RAPD types with average genetic similarity of $33.25 \%(66.75 \%$ average genetic diversity). The level of similarity ranged between $23.7 \%$ and $42.8 \%$. The genetic similarity between serotype Salmonella Lindenburg and Salmonella Enteritidis was 48.55\%

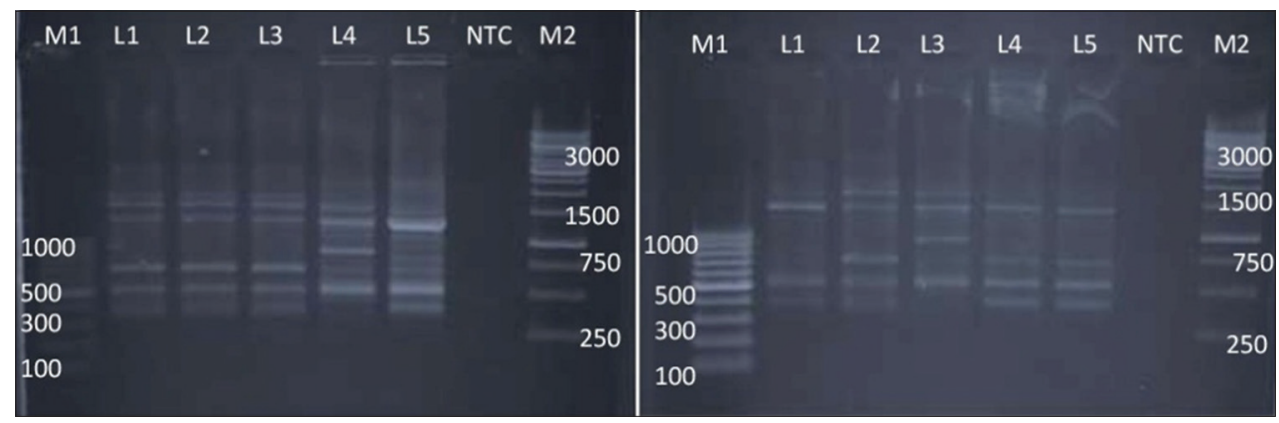

Figure-7: Random amplified polymorphic DNA - polymerase chain reaction profiles of Salmonella enterica isolates with NSC II. Plate 1- Lane M1: 100 bp ladder; Lane 1: Salmonella Lindenburg; Lane 2: Salmonella Lindenburg; Lane 3: Salmonella Lindenburg; Lane 4: Salmonella Rough; Lane 5: Salmonella Rough; Lane 6: NTC; Lane M2: 1 kb ladder and Plate 2 Lane M1: 100 bp ladder; Lane 1: Salmonella Enteritidis Lane 2: Salmonella Lindenburg; Lane 3: Salmonella Rough; Lane 4: Salmonella Rough; Lane 5: Salmonella Typhimurium; Lane 6: NTC; Lane M2: 1 kb ladder.

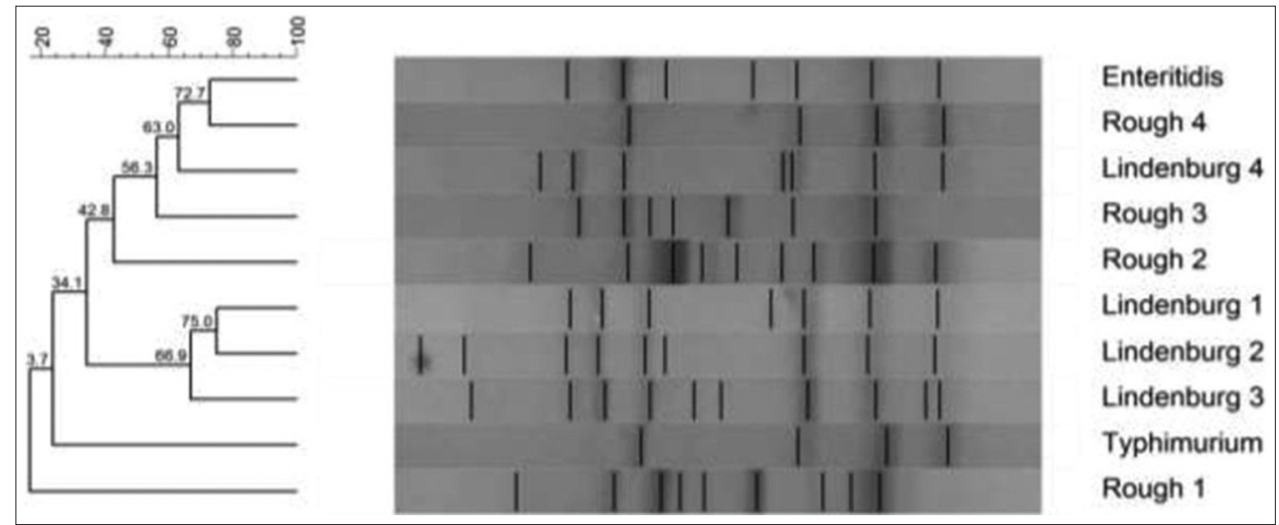

Figure-8: Dendrogram of random amplified polymorphic DNA - polymerase chain reaction profile of Salmonella enterica isolates with NSC II. 
(51.45\% average genetic diversity). The level of similarity ranged between $34.1 \%$ and $63 \%$. The genetic similarity between serotype Salmonella Lindenburg and Salmonella Typhimurium was 34.1\% (65.9\% average genetic diversity). The genetic similarity between serotype Salmonella Enteritidis and Salmonella Typhimurium was $34.1 \%$ (65.9\% average genetic diversity).

The genetic similarity among Salmonella spp. from chicken ranged between $42.8 \%$ and $75 \%$. The average similarity was $61.56 \% \quad(38.44 \%$ average genetic diversity) and for those isolates from milk ranged from $34.1 \%$ to $56.3 \%$ with an average similarity of $45.2 \%$ (54.8\% average genetic diversity). Average genetic similarity between Salmonella spp. isolated from egg was $42.8 \%$ (57.2\% average genetic diversity) and from egg and chicken was $48.55 \%$ (51.45\% average genetic diversity). The discrimination power of NSC II was 0.1842 . A report by Rezk et al. [27] showed maximum genetic variability among Salmonella Paratyphi B isolates using NSC II primer when compared to NSC I and NSC III.

Similarly, a study by Meenu [28] showed maximum genetic variability among Salmonella Gallinarum isolates of poultry origin using NSC II primer when compared to NSC I and 1290. Sumithra [26] also reported that D value of NSC II primer was better than 1290 similar to previous results, but it was similar to that of NSCI for the heterogeneity study of Salmonella Typhimurium. However, on the contrary to previous reports, this study showed that the discriminative power of NSC I primer is better than NSC II for differentiation of different Salmonella serovars. This variation may be due to the difference in reaction mixture such as the amount of magnesium chloride used in the reaction mixture. Also, difference in serotype and origin of the isolates may also be taken into consideration.

RAPD-PCR using primer 3 was also able to produce fingerprints of all the 10 isolates of Salmonella spp. tested (Figure-9). On the basis of dendrogram prepared using RAPD-PCR assay, 10 isolates of Salmonella could be divided into 10 RAPD-PCR types (Figure-10). The analysis of fingerprints in the dendrogram of four isolates of Salmonella Lindenburg showed four different types of fingerprints, hence, dividing them into four clonal groups. We observed an average genetic similarity between four isolates of Salmonella Lindenburg as 33.7\% (66.3\% average genetic diversity). The level of similarity ranged from $13.6 \%$ to $66.7 \%$. The four Salmonella Rough strains were separated into four different RAPD types with average genetic similarity of $43.75 \%$ (56.25\% average genetic diversity). The genetic similarity between serotype Salmonella Lindenburg and Salmonella Enteritidis was $21.8 \%(78.2 \%$ average genetic diversity) and between serotype Salmonella Lindenburg and Salmonella Typhimurium was $17.2 \%$ (82.8\% average genetic diversity). The genetic similarity between serotype Salmonella Enteritidis and

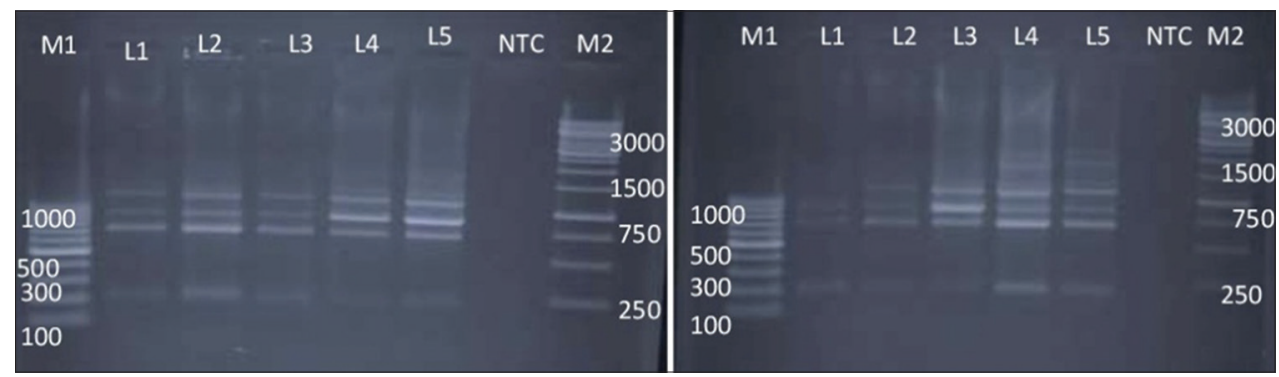

Figure-9: Random amplified polymorphic DNA - polymerase chain reaction profiles of Salmonella enterica isolates with primer 3. Plate 1 - Lane M1: 100 bp ladder; Lane 1: Salmonella Lindenburg; Lane 2: Salmonella Lindenburg; Lane 3: Salmonella Lindenburg; Lane 4: Salmonella Rough; Lane 5: Salmonella Rough; Lane 6: NTC; Lane M2: 1 kb ladder and Plate 2 - Lane M1: 100 bp ladder; Lane 1: Salmonella Enteritidis; Lane 2: Salmonella Lindenburg; Lane 3: Salmonella Rough; Lane 4: Salmonella Rough; Lane 5: Salmonella Typhimurium; Lane 6: NTC; Lane M2:1 kb ladder.

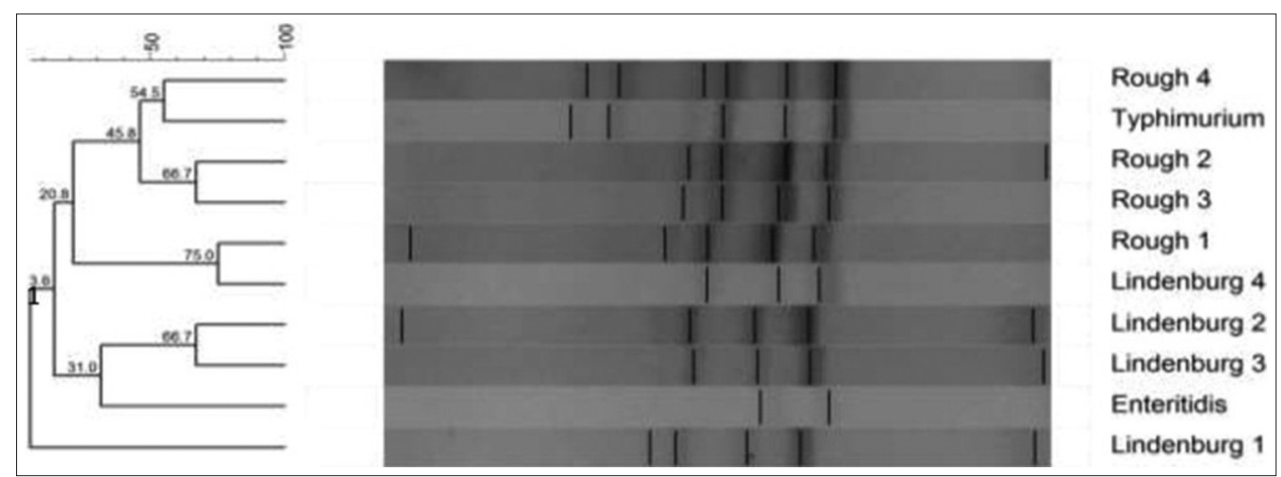

Figure-10: Dendrogram of random amplified polymorphic DNA - polymerase chain reaction profile of Salmonella enterica isolates with primer 3. 
Salmonella Typhimurium was $13.6 \%$ (86.4\% average genetic diversity).

Salmonella spp. from chicken showed a genetic similarity ranged between $13.6 \%$ and $66.7 \%$ with an average similarity of $33.7 \%(66.3 \%$ average genetic diversity) and those milk ranged from 45.8 to $54.8 \%$ with an average of $50.15 \%$ (49.85\% average genetic diversity). Average genetic similarity between Salmonella spp. isolated from egg was 20.8\% (79.2\% average genetic diversity) and from egg and chicken is $25.9 \%$ ( $74.1 \%$ average genetic diversity). The discrimination power of primer 3 was 0.2582 .

Due to polymorphism inherent in the sequence, as well as, the short length of primers used, the RAPD typing method resulted in a clustering of isolates into highly discriminating genetic trees [27]. Although relatively few samples were used in the study, the data suggest that RAPD typing is discriminatory; it is easy to interpret and constitute a low-cost method to type the various Salmonella serovars. These observations revealed that RAPD profile could be best used for finding out the heterogeneity at the molecular level of Salmonella isolates in combination with other molecular and phenotypic typing techniques. Thus, our results support the earlier observation of its significance by different workers on different Salmonella serotypes [28-35].

\section{Conclusion}

The high discriminative power of NSCI revealed the power of this primer as a potential candidate in RAPD analysis of Salmonella isolates, and further, it can be used for the epidemiological studies for the classification of Salmonella isolates based on their geographical origin. However, still, it has demerit due to its non-reproducibility. It once again proved that repeatability of RAPD-PCR is insufficient to distinguish genetic differences among Salmonella serovars.

\section{Authors' Contributions}

SSS carried out the research work; AG planned, designed, and supervised the experiment; BJ assisted in planning designing and execution of work. HK and NK assisted in the collection of sample and laboratory work. All authors read and approved the final manuscript.

\section{Acknowledgments}

The authors are thankful to the Vice Chancellor RAJUVAS, Bikaner and Dean CVAS, Navania, Udaipur, Rajasthan, India for providing all necessary laboratory facilities and chemicals for the research. The authors declare that they did not receive any funding source or grant to support this research work.

\section{Competing Interests}

The authors declare that they have no competing interests.

\section{Publisher's Note}

Veterinary World remains neutral with regard to jurisdictional claims in published institutional affiliation.

\section{References}

1. Manoj, J., Singh, M.K. and Singh, Y.P. (2015) The role of poultry in foodborne salmonellosis and it's public health importance. Adv. Anim. Vet. Sci., 3(9): 485-490.

2. Barrow, P.A. and Methner, U. (2013) Salmonella in domestic animals. CABI, UK.

3. Painter, J.A., Hoekstra, R.M., Ayers, T., Tauxe, R.V., Braden, C.R., Angulo, F.J., Griffin, P.M. (2013) Attribution of foodborne illnesses, hospitalizations, and deaths to food commodities by using outbreak data, United States, 19982008. Emerg. Infect. Dis., 19(3): 407-415.

4. Antunes, P., Mourao, J., Campos, J. and Peixe, L. (2016) Salmonellosis: The role of poultry meat. Clin. Microbiol. Infect., 22(2): 110-121.

5. Obaro, S.K., Tam, P.Y.I. and Mintz, E.D. (2017) The unrecognized burden of typhoid fever. Expert Rev. Vaccin., 16(3): 249-260.

6. Hernandeza, M., Gomez, J., Luqueb, I., Herrera, S., Maldonadob, A., Reguillob, L. and Astorgab, R.J. (2013) Salmonella prevalence and characterization in a free-range pig processing plant: Tracking in trucks, lairage, slaughter line and quartering. Int. J. Food Microbiol., 162(1): 48-54.

7. Who, P.Y., Thong, K.L., Behnke, J.M., Lewis, J.W. and Zain, S.N.M. (2017) Characterization of nontyphoidal Salmonella isolates from asymptomatic migrant food handlers in Peninsular Malaysia. J. Food Prot., 80(8): 1378-1383.

8. Dobhal, S., Zhang, G., Rohla, C., Smith, M.W. and Ma, L.M. (2014). A simple, rapid, cost-effective, and sensitive method for detection of Salmonella in environmental and pecan samples. J. Appl. Microbiol., 117(4): 1181-1190.

9. Parmley, E.J., Pintar, K., Majowicz, S., Avery, B., Cook, A., Jokinen, C., Gannon, V., Lapen, D.R., Topp, E., Edge, T.A., Gilmour, M., Pollari, F., Reid-Smith, R. and Irwin, R. (2013) A Canadian application of one health: Integration of Salmonella data from various Canadian surveillance programs (2005-2010). Foodborne Pathog. Dis., 10(9): 747-756.

10. Martínez-Gamboa, A., Silva, C., Fernández-Mora, M., Wiesner, M., de León, A.P. and Calva, E. (2015) IS200 and multilocus sequence typing for the identification of Salmonella enterica serovar Typhi strains from Indonesia. Int. Microbiol., 18(2): 99-104.

11. Sankar, S., Kuppanan, S., Nandagopal, B. and Sridharan, G. (2013) Diversity of Salmonella enterica serovar Typhi strains collected from India using variable number tandem repeat (VNTR)-PCR analysis. Mol. Diagn. Ther., 17(4): 257-264.

12. Tau, N.P., Smith, A.M., Wain, J.R., Tarupiwa, A., Coulibaly, K.J., Keddy, K.H. and Germs-Sa (2017) Development and evaluation of a multiple-locus variable-number tandem-repeats analysis assay for subtyping Salmonella Typhi strains from sub-Saharan Africa. J. Med. Microbiol., 66(7): 937-945.

13. Silva, C., Betancor, L., García, C., Astocondor, L., Hinostroza, N., Bisio, J., Rivera, J., Perezgasga, L., Escanda, V.P., Yim, L., Jacobs, J. and Portillo, F.G. (2017) Characterization of Salmonella enterica isolates causing bacteremia in Lima, Peru, using multiple typing methods. PLoS One, 12(12): e0189946.

14. Barco, L., Barrucci, F., Olsen, J.E. and Ricci, A. (2013) Salmonella source attribution based on microbial subtyping. Int. J. Food Microbiol., 163(2-3): 193-203.

15. Yachison, C.A., Yoshida, C., Robertson, J., Nash, J.H.E., Kruczkiewicz, P., Taboada, E.N., Walker, M., Reimer, A., Christianson, S. and Nichani, A. (2017) The validation and implications of using whole genome sequencing as a replacement for traditional serotyping for a national Salmonella reference laboratory. Front Microbiol., 8: 1044.

16. Gurakan, G.C., Aksoy, C., Ogel, Z.B. and Oren, N.G. (2008) Differentiation of Salmonella Typhimurium from 
Salmonella Enteritidis and other Salmonella serotypes using random amplified polymorphic DNA analysis. Poult. Sci., 87(6): 1068-1074.

17. Shekhawat, S.S., Gaurav, A., Joseph, B., Sharma, S.K., Dadhich, R. and Vaishnava, C.S. (2018) Molecular detection of Salmonella enterica subspecies enterica serotypes in foods of animal origin by PCR. Int. J. Chem. Stud., 6(3): 3586-3590.

18. Jin, U.H., Chung, T.W., Kim, J.K., Nam, K.S., Ha, S.D. and Kim, C.H. (2000) Differentiation of Salmonella Typhimurium from Gram-negative intestinal microbes by randomly amplified polymorphic DNA (RAPD) fingerprinting. J. Microbiol., 38(1): 8-10.

19. Habtamu, T.M., Rathore, R., Dhama, K. and Agarwal, R.K. (2011) Epidemiological characterization of Salmonella Gallinarum isolates of poultry origin in India, employing two PCR based typing methods of RAPD-PCR and PCRRFLP. Asian J. Anim. Vet. Adv., 6(11): 1037-1051.

20. Aksoy, C. (2004). Identification of Serotype-Specific DNA marker for Salmonella Typhimurium by RAPDPCR Method. A Thesis Submitted to the Graduate School of Natural and Applied Sciences of Middle East Technical University in Partial Fulfillment of the Requirements for the Degree of Master of Science in Biotechnology.

21. Hunter, P.R. and Goston, M.A. (1988) Numerical index of the discriminatory ability of typing systems: An application of Simpson's index of diversity. J. Clin. Microbiol., 26(11): 2465-2466.

22. Welsh, J. and McClelland, M. (1990) Fingerprinting genomes using PCR with arbitrary primers. Nucleic Acids Res., 18(24): 7213-7218.

23. Williams, J.G.K., Kubelik, A.R., Livak, K.J., Rafalski, J.A. and Tingey, S.V. (1990) DNA polymorphisms amplified by arbitrary primers are useful as genetic markers. Nucleic Acids Res., 18(22): 6531-6535.

24. Caetano-Anolles, G., Bassam, B.J. and Gresshoff, P.M. (1991) DNA amplification fingerprinting using very short arbitrary oligonucleotide primers. Biotechnology, 9(6): 553-556.

25. Sumithra, T.G., Chaturvedi, V.K., Gupta, P.K., Patel, C.L., Joseph, B., Rai, A.K., Sunita, S.C. and Kollannur, J.D.
(2013) Application of random amplified polymorphic DNA analysis in discrimination of Salmonella Typhimurium isolates of India. J. Vet. Healthc., 11(1): 43-50.

26. Sumithra, T.G. (2010) Studies on Molecular Heterogeneity Among Salmonella Typhimurium Isolates of Diverse Origin. M. V. SC. Thesis Submitted to Deemed University, IVRI, Izzatnagar, U.P., India.

27. Rezk, N.A., Mansour, H., Ghoneim, N.H. and Rifaat M.M. (2012) Typing of Salmonella Typhi strains isolated from Egypt by RAPD PCR. 3 Biotech., 2(1): 17-25.

28. Meenu, A. (2002) Study of Heterogeneity in Indian Isolates of S. Paratyphi B. M. V. SC Thesis Submitted to Deemed University, IVRI., Izatnagar, U.P., India.

29. Menghistu, H.T. (2009) Studies on Molecular Heterogeneity Among Salmonella Gallinarum Isolates of Poultry Origin. M. V. SC. Thesis Submitted to Deemed University, IVRI, Izatnagar, U.P., India.

30. Wang, L., Shi, L., Alam, M.J., Geng, Y. and Li, L.(2008) Specific and rapid detection of foodborne Salmonella by loop-mediated isothermal amplification method. Food Res. Int., 41(1): 69-74.

31. Fadl, A., Nguyen, A.V. and Khan, M.I. (1995) Analysis of Salmonella Enteritidis isolates by arbitrarily primed PCR. $J$. Clin. Microbiol., 33(4): 987-989.

32. Skibsted, U., Baggesen, D.L., Dessau, R. and Lisby, G. (1998) Random amplification of polymorphic DNA (RAPD), pulsed-field gel electrophoresis (PFGE) and phage-typing in the analysis of a hospital outbreak of Salmonella Enteritidis. J. Hosp. Infect., 38(3): 207-216.

33. Mhand, R.A., Brahimi, N., Moustaoui, N., Mdaghri, N., Amarouch, H., Grimont, F., Bingen, E. and Benbachir, M. (1999) Characterization of extended-spectrum-lactamase-producing Salmonella Typhimurium by phenotypic and genotypic typing methods. J. Clin. Microbiol., 37(11): 3769-3773.

34. Saxena, M.K., Singh, V.P., Saxena, A., Lakhchaura, B.D. and Sharma, B. (2003) Strain differentiation of Salmonella Bareilly by AP-PCR. Indian J. Biotechnol., 2: 607-609.

35. Verma, J.K. (2009). Isolation and Molecular Characterization of Poultry Isolates of Salmonella Typhimurium. M. V. SC. Thesis Submitted to the G.B. Pant University of Agriculture and Technology, Pantnagar, Uttarakhand., India. 